\title{
Endodontic treatment of a mandibular premolar with Vertucci type $V$ root canal morphology: a rare case report
}

\author{
Yelda Erdem Hepşenoğlu, Şeyda Erşahan \\ Department of Endodontics, İstanbul Medipol University Faculty of Dentistry, İstanbul, Turkey
}

\begin{abstract}
Mandibular premolars have been reported with complex anatomical aberrations, making endodontic treatment of these teeth extremely difficult. A case of endodontic retreatment of a mandibular second premolar exhibiting root canal bifurcation (Vertucci type $V$ root canal configuration) of two apical foramina was described. A 39-year-old female with a non-significant medical history presented with a chief complaint of pain in a previously endodontically treated tooth. Clinical examination revealed tenderness to percussion. Intraoral periapical radiographs taken at different angulations revealed one root and two apical foramina. The main root canal was filled short of the apex and the other canal was not filled due to the broken file. Root canal retreatment was performed following the standard protocols. Although the prevalence of one root and two apical foramina in mandibular premolar is very low, clinicians should always be aware of variations in the number of roots and canals for the proper management of such cases.
\end{abstract}

Keywords: Broken file; mandibular premolar; morphologic variation; retreatment; Vertucci type $\mathrm{V}$ root canal.

\begin{abstract}
$\mathrm{A}$ thorough understanding of the root canal morphology and correct evaluation of preoperative radiographs are essential for endodontic treatment. As many cases of endodontic treatment failure are due to missed canals, clinicians should be aware of all types of variations that may exist in the root canal system of each tooth. ${ }^{[1]}$ Vertucci $^{[2,3]}$ (2005) classified morphological patterns of the root canal systems into eight types. In the simplest form, each root has a single canal and a single apical foramen (type I). However, other canal complexities that exit the root as one, two, or more apical canals (types II-VIII) exist. ${ }^{[2,3]}$ In general, the mandibular second premolars have a single root and a single canal (Vertucci type I; $99.28 \%$ and $86.9 \%$, respectively). ${ }^{[4]}$ However, the incidence of a single root with two
\end{abstract}

canals (Vertucci type $\mathrm{V}$ ) in the mandibular second premolars is very rare $(6.4 \%-29 \%) .^{[5,6]}$ Treatment failures and post-treatment flare-ups have been reported in these teeth, thus indicating too much variation in the morphology of mandibular second premolars. ${ }^{[7,8]}$ To date, only a few retreatment cases of mandibular second premolars with one root and two canals have been reported in the literature. ${ }^{[9]}$ This case report describes the successful retreatment of an unusual mandibular second premolar with Vertucci type $\mathrm{V}$ root canal morphology.

\section{Case report}

A 39-year-old female patient was referred by her general dentist to the Istanbul Medipol University, Faculty of

Correspondence: Dr. Şeyda Erşahan. İstanbul Medipol Üniversitesi Diş Hekimliği Fakültesi Hastanesi,

Birlik Mah., Bahçeler Cad., No: 5, Esenler, İstanbul, Turkey.

Tel: +90 212 - 4401000 e-mail: seydaersahan@hotmail.com

Submitted: March 12, 2018 Accepted: April 27, 2018 
Dentistry, Department of Endodontics, for retreatment of her right mandibular second premolar. Patient's medical history was non-significant. The tooth was moderately sensitive to percussion and exhibited normal mobility. Preoperative radiographic examination revealed one root with two canals and only one root canal was filled; the other canal was not entered due to the broken file at the level of the middle third of the root, suggesting a Vertucci type $\mathrm{V}$ configuration. Radiographic appearance of the periapical region was normal (Fig. 1). Based on these findings, a diagnosis of symptomatic apical periodontitis was made and root canal retreatment was recommended. Endodontic access cavity was prepared after injection of a local anesthetic and rubber dam isolation was performed (40 $\mathrm{mg}$ articaine hydrochloride with $0.006 \mathrm{mg} / \mathrm{mL}$ epinephrine hydrochloride; Ultracaine DS forte; Aventis Pharma, Istanbul, Turkey). After gaining access to the previously obturated buccal root canal, \#1, \#2, and \#3 Gates Glidden burs (Mani Inc., Tochigi, Japan) were used to coronal two-thirds of the buccal canal, whereas an ISO size \#15 K-

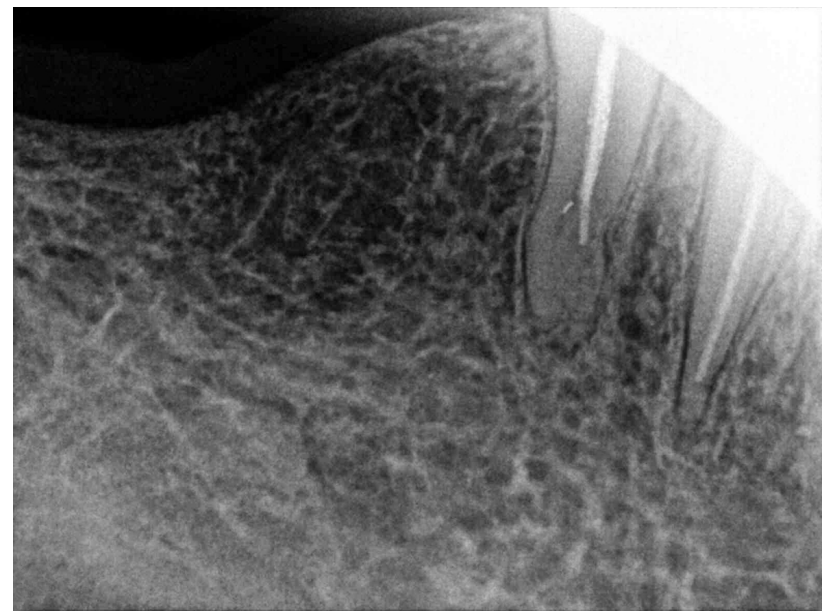

Fig. 1. Diagnostic radiograph showing root canal bifurcation.

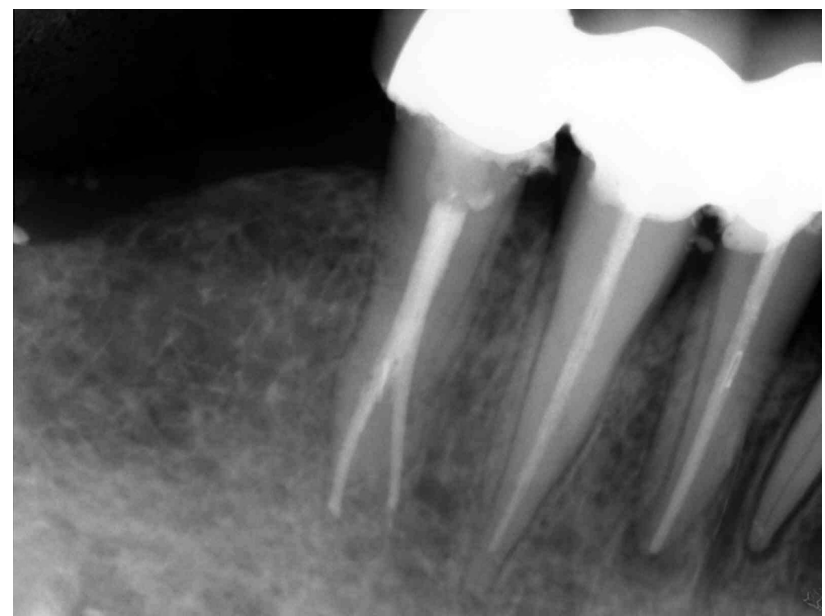

Fig. 2. Periapical radiograph following obturation of the root canals. file (Dentsply-Maillefer, Ballaigues, Switzerland) was used to gain access to the apical third of this canal. An attempt was made to bypass and retrieve the previously fractured fragment using a \#10 K-file. Then, the working length was determined using an apex locator (Raypex 6; VDW, Munich, Germany) and later confirmed with radiography. The crown-down technique was performed for cleaning and shaping using Revo S files (Micro-Mega, Besançon, France) and 5\% sodium hypochlorite irrigation (\#40/.06 taper and \#25/.04 taper for buccal and lingual canals, respectively). Patency was achieved in all the canals and was maintained using a \#10 K-file. A final working-length periapical radiograph showed two canals located by gutta percha cones (Fig. 2). Ethylenediaminetetraacetic acid (17\%) and saline were used for final irrigation (Wizard; Rehber Chemistry, Istanbul, Turkey). The root canals were dried with paper points and obturated with laterally condensed gutta percha cones (AH Plus, Dentsply, DeTrey, Konstanz, Germany). A postoperative periapical radiograph showed complete filling of the two bifurcated canals (Fig. 2 ). The root canal retreatment was completed in a single visit. The tooth was then restored with composite resin (3M, St. Paul, MN, USA) and the patient was followed up after 1 month. Following the retreatment of the right mandibular second premolar, the patient, who felt no obvious symptoms or signs, was referred back to her general dentist for prosthetic treatment. According to her general dentist, the tooth was free of palpation pain, percussion pain, and biting pain after the prosthetic procedure.

\section{Discussion}

The complex anatomy of the mandibular premolar should be thoroughly understood for proper management and better prognosis. ${ }^{[10]}$ Vertucci type V root canal morphology in mandibular premolars has great clinical importance during endodontic treatment. Poor or no filling of the lingual root canal in this morphology, in which there are two independent apical foramina, may lead to failure of the endodontic treatment. ${ }^{[11]}$ The presence of an untreated canal can result in persistent symptoms and/or signs, such as pain and thickening of the periodontal ligament space. ${ }^{[12]}$ In this patient, we successfully treated a mandibular second premolar with a Vertucci type V root canal morphology. Several studies regarding anatomical variations in mandibular premolars are available in the literature. ${ }^{[4-6]}$ In both mandibular first and second premolars, single canals are most likely to be an independent canal from the orifice to the apical foramen (type I) (81\% and $90 \%$, respectively, for mandibular first and second premolars) ${ }^{[4]}$ However, a significant number of bifid canals fuse prior to exit (type II) in mandibular first premolars of Indian and Turkish 
populations $(9 \%-18 \%) .^{[5,13-15]}$ Type IV pattern is more prevalent than type $\mathrm{V}$ pattern in most population groups. In second premolars, type $\mathrm{V}$ pattern is more prevalent than type IV in all population groups, with significantly higher prevalence of up to $15 \%-17 \%$ in Indian and Jordanian populations. ${ }^{[4]}$ Genetic and racial variations are factors that may affect root canal anatomy and morphology. In a Turkish population, Sert and Bayirli ${ }^{[6]}$ evaluated the proportion of different root canal types, and they reported that $6 \%-8 \%$ of mandibular second premolars had Vertucci type $\mathrm{V}$ root canal morphology.

Intraoral periapical radiographs are essential for identifying the internal anatomy of the tooth. Radiographs taken at different horizontal angulations helps in better visualization of the canals. In the present case, the canal bifurcated into two and the division was buccal and lingual, with the lingual canal splitting from the main canal at a sharp angle and in which a broken file fragment was present. As we observed canal divergence with a standard periapical radiograph, we did not need to take an angled radiograph or cone beam computed tomography.

In addition, the bifurcation point of the two canals was at the apical third of the root. Therefore, it was a challenge to negotiate the second canal at the apical third. Slowey et al. ${ }^{[16]}$ recommends visualizing this canal configuration as a lower case letter "h." In the present case, the buccal canal was the straight-line portion of the letter "h," whereas the lingual canal existed below the mid-root at a sharp angle from the straight buccal canal, as indicated by Slowey et al. The shape of the access cavity was ovoid in the buccolingual direction. Tactile examination of all the walls of the major canal with the tip of a small, precurved scouting K-file was used to indicate the orifice of the lingual canal, and the lingual canal was manually reproduced. If this method fails to find the orifice, alternative methods could be used as follows: troughing of the grooves with ultrasonic tips, staining the chamber floor with $1 \%$ methylene blue dye, performing the sodium hypochlorite "champagne bubble test," and visualizing canal bleeding points, with the use of microscopes, magnifying loupes, and fiber-optic transillumination.

First, we attempted to bypass and retrieve the previously fractured fragment using a \#10 K-file. Then, we established a glide path before commencing the cleaning and shaping. Preventing further file fracture was accomplished with the use of fresh instruments (only one of \#08 and \#10 files each), frequent inspection of files for distortion, precurving hand files, use of lubricant, and use of flexible Ni-Ti rotary and hand files. The lingual canal splitting from the main canal at a sharp angle was prepared to a smaller apical size and taper (\#25/.04) than that of the buccal canal $(\# 40 / .06)$. Clinician should be careful in the instrumentation of such canals, as overzealous instrumentation can lead to strip or lateral perforations in these critical areas. Obturation of Vertucci type V root canal morphology requires specific technique modifications. Adequate coronal canal flaring was used to access the bifurcated canal orifices, and as the coronal space was insufficient to accommodate two master cones simultaneously, the larger master cone was cut extraorally at the level of the bifurcation or orifice. This shortened master cone was then spread at the blunt, cut end using a spreader in clockwise direction. Care was taken to prevent blocking of the lingual canal using a sealer or remnant gutta percha by placing a paper point within the canal that was to be filled later. The remaining canal was then obturated similarly. Then, backfilling of the coronal portion of the canal was performed. Alternatively, such root canal systems can also be obturated using the "Squirt technique," ${ }^{[17,18]}$ in which injection of a thermoplasticized gutta percha is followed by backfilling.

Mandibular premolars, because of their complex canal systems, are often considered the most difficult of all teeth for performing successful endodontic treatment. ${ }^{[6,16]}$ However, various innovations in diagnostics, magnification, operative instruments, and techniques and an updated knowledge of the anatomy of mandibular premolars could certainly improve the endodontic success rates of even the most challenging cases.

\section{Conclusion}

Mandibular premolars are undoubtedly an endodontic challenge because of the presence of extra root canals, which may be more common than expected. This case report highlights the importance of having a thorough knowledge of the root canal anatomy, careful interpretation of radiographs, proper modification of the conventional access cavity for recognition, and adequate treatment of these anatomical variations.

Conflict of interest: None declared.

\section{References}

1. Ferreira CM, de Moraes IG, Bernardineli N. Three-rooted maxillary second premolar. J Endod 2000;26:105-6.

2. Vertucci FJ. Root canal anatomy of the mandibular anterior teeth. J Am Dent Assoc 1974;89:369-71. [CrossRef]

3. Vertucci FJ. Root canal anatomy of the human permanent teeth. Oral Surg Oral Med Oral Pathol 1984;58:589-99.

4. Kottoor J, Albuquerque D, Velmurugan N, Kuruvilla J. Root anatomy and root canal configuration of human permanent mandibular premolars: a systematic review. Anat 
Res Int 2013;2013:254250. [CrossRef]

5. Calişkan MK, Pehlivan Y, Sepetcioğlu F, Türkün M, Tuncer SS. Root canal morphology of human permanent teeth in a Turkish population. J Endod 1995;21:200-4.

6. Sert S, Bayirli GS. Evaluation of the root canal configurations of the mandibular and maxillary permanent teeth by gender in the Turkish population. J Endod 2004;30:3918. [CrossRef]

7. Hargreaves KM, Cohen S, Berman LH, editors. Cohen's Pathways of the Pulp. 10th ed. St Louis: Mosby Elsevier; 2011.

8. Glassman GD. Flare-up with associated paresthesia of a mandibular second premolar with three root canals. Oral Surg Oral Med Oral Pathol Oral Radiol Endod 1987;64: 110-3. [CrossRef]

9. Shokouhinejad N. Root canal re-treatment of a mandibular second premolar with three root canals: a case report. Aust Endod J 2009;35:180-2. [CrossRef]

10. Pineda F, Kuttler Y. Mesiodistal and buccolingual roentgenographic investigation of 7,275 root canals. Oral Surg Oral Med Oral Pathol 1972;33:101-10. [CrossRef]

11. Yoshioka T, Villegas JC, Kobayashi C, Suda H. Radiographic evaluation of root canal multiplicity in mandibular first premolars. J Endod 2004;30:73-4. [CrossRef]

12. Fan B, Cheung GS, Fan M, Gutmann JL, Bian Z. Cshaped canal system in mandibular second molars: Part IAnatomical features. J Endod 2004;30:899-903. [CrossRef]

13. Parekh V, Shah N, Joshi H. Root canal morphology and variations of mandibular premolars by clearing technique: an in vitro study. J Contemp Dent Pract 2011;12:318-21.

14. Jain A, Bahuguna R. Root canal morphology of mandibular first premolar in a gujarati population - an in vitro study. Dent Res J (Isfahan) 2011;8:118-22.

15. Sikri VK, Sikri P. Mandibular premolars: aberrations in pulp space morphology. Indian J Dent Res 1994;5:9-14.

16. Slowey RR. Root canal anatomy. Road map to successful endodontics. Dent Clin North Am 1979;23:555-73.

17. Hermann HW, Hülsmann M. Problems of root canal obturation. In: Hülsmann M, Schäfer E, editors. Problems in Endodontics-Etiology, Diagnosis and Treatment. 1st ed. London: Quintessence Publishing; 2009. p. 293-333.

18. da Silva D, Endal U, Reynaud A, Portenier I, Orstavik D, Haapasalo M. A comparative study of lateral condensation, heat-softened gutta-percha, and a modified master cone heat-softened backfilling technique. Int Endod J 2002;35:1005-11. [CrossRef] 